\title{
Treadmill exercise ameliorates impairment of spatial learning memory in pups born to old and obese mother rats
}

\author{
Sang-Hoon Kim',2, II-Gyu Ko ${ }^{2}$, Jun-Jang Jin², Lakkyong Hwang², Seung-Soo Baek ${ }^{1, *}$ \\ 'Department of Sport \& Health Sciences, College of Art \& Culture, Sangmyung University, Seoul, Korea \\ ${ }^{2}$ Department of Physiology, College of Medicine, Kyung Hee University, Seoul, Korea
}

Memory state of rat pups born to old and obese mother rats and the effect of a treadmill running of mother rats on the memory of rat pups were studied. The radial 8-arm maze test was performed to detect spatial learning memory, and the level of tumor necrosis factor- $\alpha$, interleukin (IL)-1 $\beta$, and IL- 6 in the hippocampus was measured by enzymelinked immunoassay. Western blotting was performed for the expression of nuclear factor kappa-light-chain-enhancer (NF- $\kappa B$ ), nuclear factor of kappa light polypeptide gene enhancer in B-cells inhibitor, alpha (IKB- $\alpha$ ), B-cell lymphoma 2 (Bcl-2), Bcl-2-associated X protein (Bax), matrix metalloproteinase (MMP)-9, and immunohistochemistry for caspase- 3 was conducted. The newborn rats were classified into following groups: pups born to old mother rats, pups born to old mother rats with exercise, pups born to old and obese mother rats, and pups born to old and obese mother rats with exercise. Exercise of mother ameliorated spatial learning memory impairment, inhibited proinflammatory cytokines production, NF- $\kappa B$ expression, and $I_{\kappa} B-\alpha$ phosphorylation of the pups born to old and obese mother rats. Maternal exercise suppressed Bax expression, the number of caspase-3, the level of MMP-9, and enhanced Bcl-2 expression of the pups born to old and obese mother rats. When the maternal exercise was performed, the impairment of spatial learning memory in pups was ameliorated. Therefore, it can be seen that exercise during pregnancy of older and obese mothers is an important factor in fetal health management.

Keywords: Spatial learning memory, Treadmill exercise, Apoptosis, Nuclear factor kappa-light-chain-enhancer, Matrix metalloproteinase-9

\section{INTRODUCTION}

Children born to obese parents have a high rate of becoming obese and have a high probability of developing various diseases such as heart disease, cancer, and high blood pressure. It is known that excessive nutritional intake of the mother affects abnormal neural circuit growth in the fetus and changes in the sensitivity of leptin in the hypothalamus, which impairs the viability of progenitor cells in the hippocampus, thereby reducing the neuronal cell production of the fetus (Chang et al., 2008). Radial 8-arm maze test is behavior test to detect spatial learning memory. Radial 8-arm maze test revealed impairment of spatial learning memory in traumatic brain injury rats. Meanwhile, treadmill exercise showed alleviation of traumatic brain injury-induced impairment of spatial learning memory in rats (Ko et al., 2019).

Nuclear factor kappa-light-chain-enhancer (NF- $\kappa$ B) is a main transcription factor of macrophages and induces several inflammatory genes, such as tumor necrosis factor- $\alpha$ (TNF- $\alpha$ ), interleukin (IL)-1 $\beta$, and IL-6 (Jeong et al., 2017). NF-kB is a protein complex that controls the transcription of DNA, cytokine production, and cell survival. NF- $\mathrm{KB}$ is associated with synaptic plasticity and memory process (Albensi and Mattson, 2000; Meffert et al., 2003). Nuclear factor of kappa light polypeptide gene enhancer in B-cells inhibitor, alpha (I $\mathrm{I} B-\alpha)$ is a member of the cellular protein family that functions to repress the NF- $\kappa \mathrm{B}$ transcription factor.

B-cell lymphoma 2 (Bcl-2) family is divided into antiapoptotic protein and proapoptotic protein according to their functions, and then determines the mitochondrial response to apoptosis stimuli
${ }^{*}$ Corresponding author: Seung-Soo Baek (iD https://orcid.org/0000-0002-1340-2098 Department of Sport \& Health Care, College of Art \& Culture, Sangmyung University, 20 Hongjimun 2-gil, Jongno-gu, Seoul 03016, Korea Email: ssoop@smu.ac.kr

Submitted: July 2, 2021 / Accepted after revision: August 1, 2021
This is an Open Access article distributed under the terms of the Creative Commons Attribution Non-Commercial License (https://creativecommons.org/licenses/by-nc/4.0/) which permits unrestricted non-commercial use, distribution, and reproduction in any medium, provided the original work is properly cited. 
(Upadhyay et al., 2003). Bcl-2 can inhibit apoptosis by inhibiting cytochrome $\mathrm{c}$ release from mitochondria. However, $\mathrm{Bcl}-2$ and $\mathrm{Bcl}-$ $\mathrm{xL}$ form heterodimers with the major proapoptotic member $\mathrm{Bcl}$-2-associated $\mathrm{X}$ protein (Bax) and lose their ability to function as a preventative (Kuwana and Newmeyer, 2003). Activation of caspases is the other important feature of apoptosis, and caspase-3 is a key performer of apoptosis (Song et al., 2018).

Proteins of the matrix metalloproteinase (MMP) family are implicated in many physiological processes and also implicated in pathological processes such as arthritis, intracerebral hemorrhage, and metastasis in the brain (Vandooren et al., 2013; Wang and Tsirka, 2005). MMP-9 performs many functions including degradation of extracellular matrix, activation of IL-1 $\beta$, and cleavage of several chemokines (Opdenakker et al., 2001).

In this experiment, the memory state of rat pups born to old and obese mother rats and the effect of a treadmill running of mother rats on the memory of rat pups were studied. The radial 8-arm maze test was performed to detect spatial learning memory, and the level of TNF- $\alpha$, IL- 6 , and IL- $1 \beta$ in hippocampus was measured by enzyme-linked immunoassay (ELISA). Western blotting was performed for the expression of NF- $\kappa \mathrm{B}$, ІкB- $\alpha$, Bax, Bcl-2, MMP9 , and immunohistochemistry for caspase- 3 was conducted.

\section{MATERIALS AND METHODS}

\section{Animals and classification}

This experiment was reviewed by the Animal Care and Use Committee of Kyung Hee University and acquired the approval number as follows: KHUASP (SE)-20-135. Old female rats were fed a normal diet ( $5 \%$ of fat), and old and obese female rats were fed a high fat diet (60\% of fat). For mating, at 46 weeks in age, Sprague-Dawley male rats (15-week-old) were reared in the same cage for 7 days at the ratio of one female and two males. Female rats were divided into two groups: one group is old mother rats $(\mathrm{n}=8)$ and the other group is old and obese mother rats $(\mathrm{n}=8)$. Treadmill running was conducted for a total of 23 weeks, including 20 weeks before and 3 weeks of pregnancy. Exercise load was at a speed of $5 \mathrm{~m} / \mathrm{min}$ for $20 \mathrm{~min}$ per day. The newborn rats were classified into following groups $(\mathrm{n}=10)$ : pups born to old mother rats, pups born to old mother rats with exercise, pups born to old and obese mother rats, and pups born to old and obese mother rats with exercise.

\section{Radial 8-arm maze test}

The radial 8-arm maze test was used to detect spatial learning memory as explained below (Ko et al., 2019). The radial arm maze device consists of a central octagonal plate and eight radial arms $35 \mathrm{~cm}$ long and $8 \mathrm{~cm}$ wide, placed $1 \mathrm{~m}$ above the floor. Cups $3 \mathrm{~cm}$ in diameter and $1 \mathrm{~cm}$ deep were placed at the ends of eight radiating arms. The rat pups were trained 3 times prior to measuring spatial learning memory. Water was forbidden for $24 \mathrm{hr}$ during training, and water was allowed to drink when found in the cup at the end of the eight radiating arms. The time it took to find the water in the cups was calculated, and the error was calculated for re-entering the arms that had already been entered, and the number of correct choices until the first error occurred was calculated. The experiment ended when the rats found water in all eight arms, or more than 8 min had passed.

\section{Enzyme-linked immunoassay}

ELISA was done to measure the level of proinflammatory cytokines such as TNF- $\alpha$, IL-6, IL- $1 \beta$, as explained Ko et al. (2020). Enzyme immunoassay kit (Abcam, Cambridge, UK) was used in accordance with the manufacturer's instructions.

\section{Western blot}

Western blot analysis for NF- $\kappa \mathrm{B}$, IкB- $\alpha$, Bax, Bcl-2, MMP-9 expression was used as explained below (Ko et al., 2020; Park et al., 2020). Hippocampal tissues were lysed in a lysis buffer containing $150 \mathrm{mM} \mathrm{NaCl}, 50 \mathrm{mM}$ Tris- $\mathrm{HCl}(\mathrm{pH}, 7.5), 1 \mathrm{mM}$ phenylmethylsulfonyl fluoride, $100-\mathrm{mg} / \mathrm{mL}$ leupeptin, $1 \%$ Nonidet $\mathrm{P} 40$, $0.5 \%$ deoxycholic acid, $0.1 \%$ sodium dodecyl sulfate. Rabbit NF$\kappa \mathrm{B}$ antibody (1:1,000; $\mathrm{Abcam})$, rabbit IкB- $\alpha$ antibody, rabbit phos-

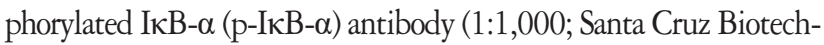
nology, Santa Cruz, CA, USA), rabbit MMP antibody (1:2,000; Cell Signaling Technology, Inc., Danvers, USA), mouse Bax antibody, mouse Bcl-2 antibody, and $\beta$-actin antibody (1:1,000; Santa Cruz Biotechnology) were used as the primary antibodies. Horseradish peroxidase-conjugated anti-mouse antibody (1:2,000; Vector Laboratories, Burlingame, CA, USA) for $\beta$-actin, Bax, Bcl-2, and horseradish peroxidase-conjugated anti-rabbit antibody (1: 2,000; Vector Laboratories) for NF- $\kappa \mathrm{B}, \mathrm{I} \kappa \mathrm{B}-\alpha$, and MMP were used as the secondary antibodies. Image-Pro Plus computer-aided image analysis system (Media Cybernetics Inc., Silver Spring, MD, USA) was used for band quantification.

\section{Immunohistochemistry}

Immunohistochemistry for capsase- 3 was done as explained below (Hwang et al., 2019; Lee et al., 2020). The sections were treated with mouse anti-caspase- 3 antibody (1:500; Santa Cruz 
Biotechnology) overnight. The sections were treated with the biotinylated mouse secondary antibody for another $1 \mathrm{hr}$. The bound secondary antibody was then amplified using a Vector Elite ABC kit (1:200; Vector Laboratories).

\section{RESULTS}

\section{Spatial learning memory}

The time to completion was increased, the number of correct choices was decreased, and the number of error choices was increased in the pups born to old and obese mother rats compared to the pups born to old mother rats. In contrast, pups born to old and obese mother rats with exercise showed decreased completion time, increased correct choice number, and decreased error choice number (Fig. 1).

\section{Proinflammatory cytokines}

Concentration of TNF- $\alpha$, IL- 6 , IL-1 $\beta$ in the hippocampus was increased in the pups born to old and obese mother rats compared to the pups born to old mother rats. In contrast, pups born to old and obese mother rats with exercise showed suppressed TNF- $\alpha$, IL-6, IL-1 $\beta$ level (Fig. 2).

\section{NF- $\kappa B$ expression and $p-1 \kappa B-\alpha$ to $1 \kappa B-\alpha$ ratio}

Expression of NF- $\kappa \mathrm{B}$ and ratio of p-IкB- $\alpha$ to $\kappa \mathrm{B}-\alpha$ in the hippocampus was increased in the pups born to old and obese mother rats compared to the pups born to old mother rats. In contrast, pups born to old and obese mother rats with exercise showed sup-

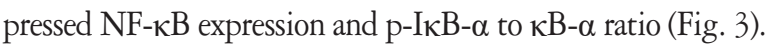

\section{Bax and $\mathrm{BCl}-2$ expression}

Expression of $\mathrm{Bax}$ in the hippocampus was increased and expression of $\mathrm{Bcl}-2$ was decreased, resulting in enhanced $\mathrm{Bax}$ to $\mathrm{Bcl}-$ 2 ratio in the pups born to old and obese maternal rats compared to the pups born to old mother rats. In contrast, pups born to old and obese mother rats with exercise showed suppressed Bax expression and enhanced $\mathrm{Bcl}-2$ expression, resulting in suppressed Bax to Bcl-2 ratio (Fig. 4).
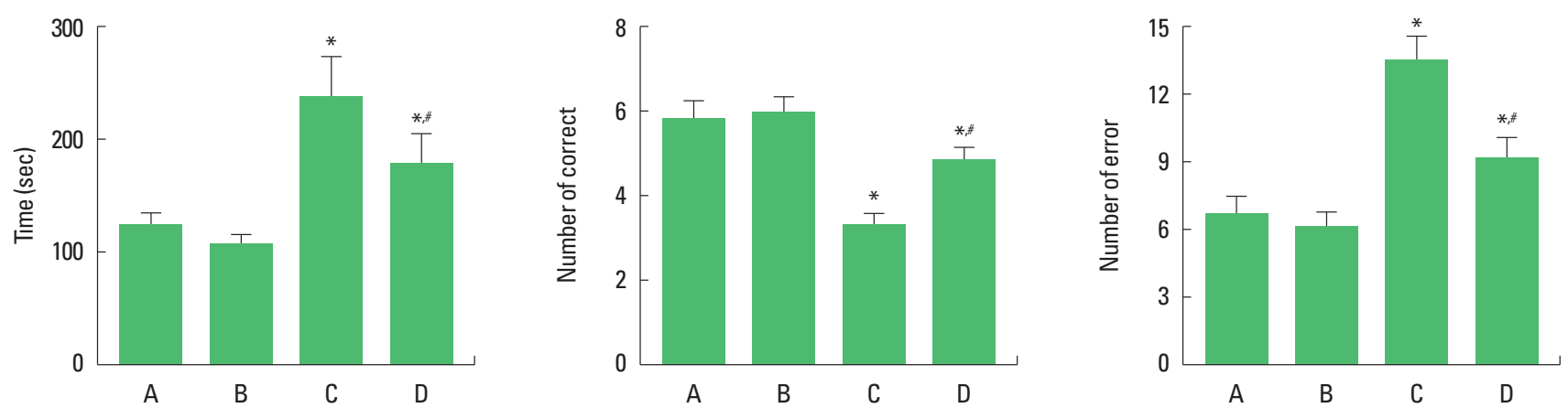

Fig. 1. Results of radial 8-arm maze test. Left panel: time to completion. Middle panel: number of correct choices. Right panel: number of error choices. A, pups born to old mother rats; $B$, pups born to old mother rats with exercise; $C$, pups born to old and obese mother rats; $D$, pups born to old and obese mother rats with exercise. ${ }^{*} P<0.05$ compared with pups born to old mother rats. ${ }^{\#} P<0.05$ compared with pups born to old and obese mother rats.
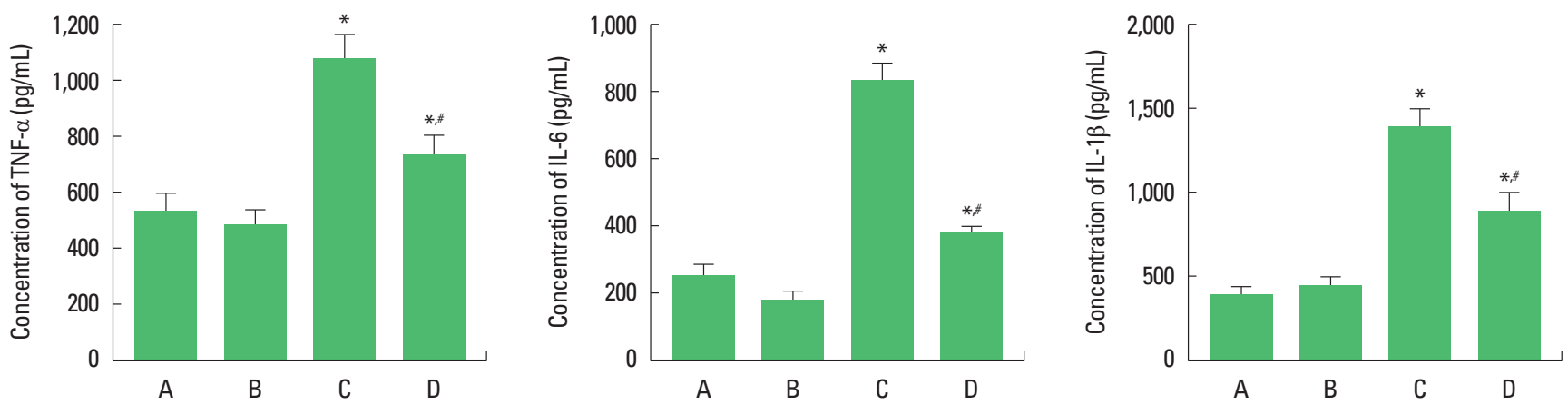

Fig. 2. Concentration of proinflammatory cytokine. Left panel: tumor necrosis factor (TNF)- $\alpha$ concentration. Middle panel: interleukin (IL)-6 concentration. Right panel: IL-1 $\beta$ concentration. $A$, pups born to old mother rats; $B$, pups born to old mother rats with exercise; $C$, pups born to old and obese mother rats; $D$, pups born to old and obese mother rats with exercise. ${ }^{*} P<0.05$ compared with pups born to old mother rats. ${ }^{\sharp} P<0.05$ compared with pups born to old and obese mother rats. 

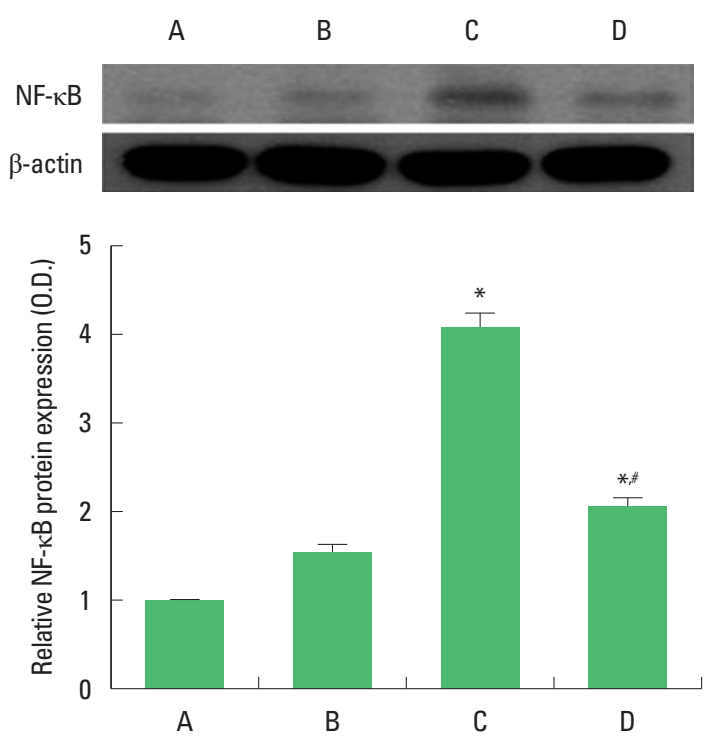
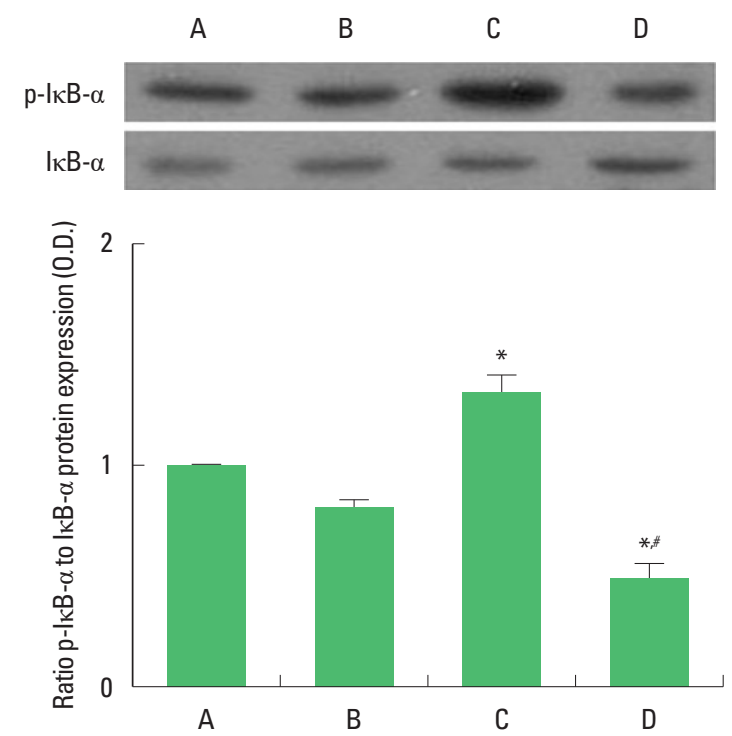

Fig. 3. Nuclear factor kappa-light-chain-enhancer (NF-kB) expression and phosphorylated nuclear factor of kappa light polypeptide gene enhancer in B-cells inhibitor,

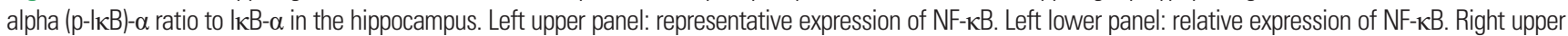
panel: representative expression of $p-\left.\right|_{\kappa} B-\alpha$ and $\mid \kappa B-\alpha$. Right lower panel: ratio of $p-l_{\kappa} B-\alpha$ and $\mid \kappa B-\alpha$. $A$, pups born to old mother rats; $B$, pups born to old mother rats with exercise; $C$, pups born to old and obese mother rats; $D$, pups born to old and obese mother rats with exercise. ${ }^{*} P<0.05$ compared with pups born to old mother rats. ${ }^{~} P<0.05$ compared with pups born to old and obese mother rats.
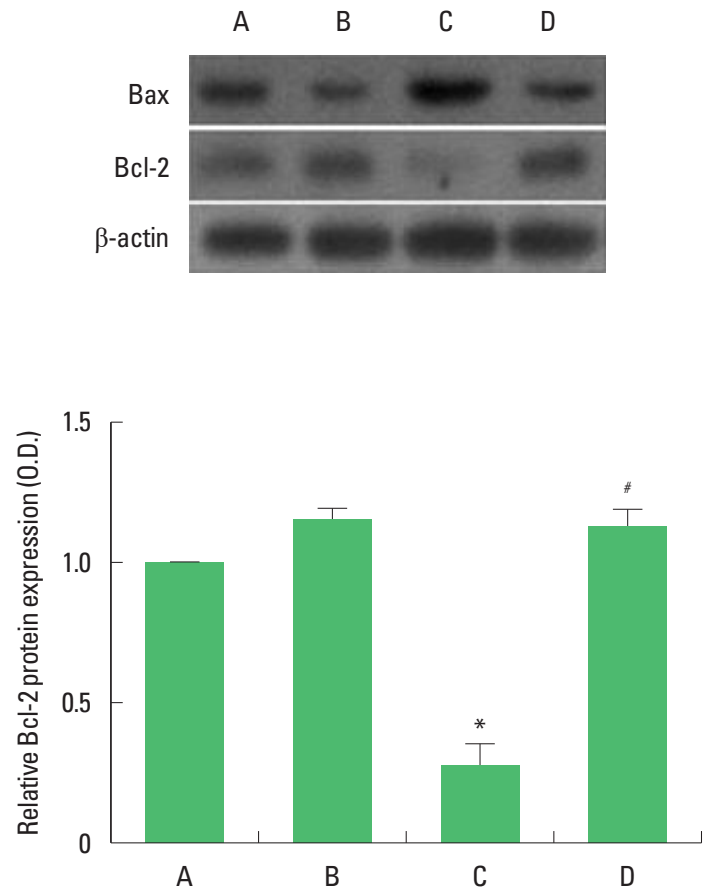
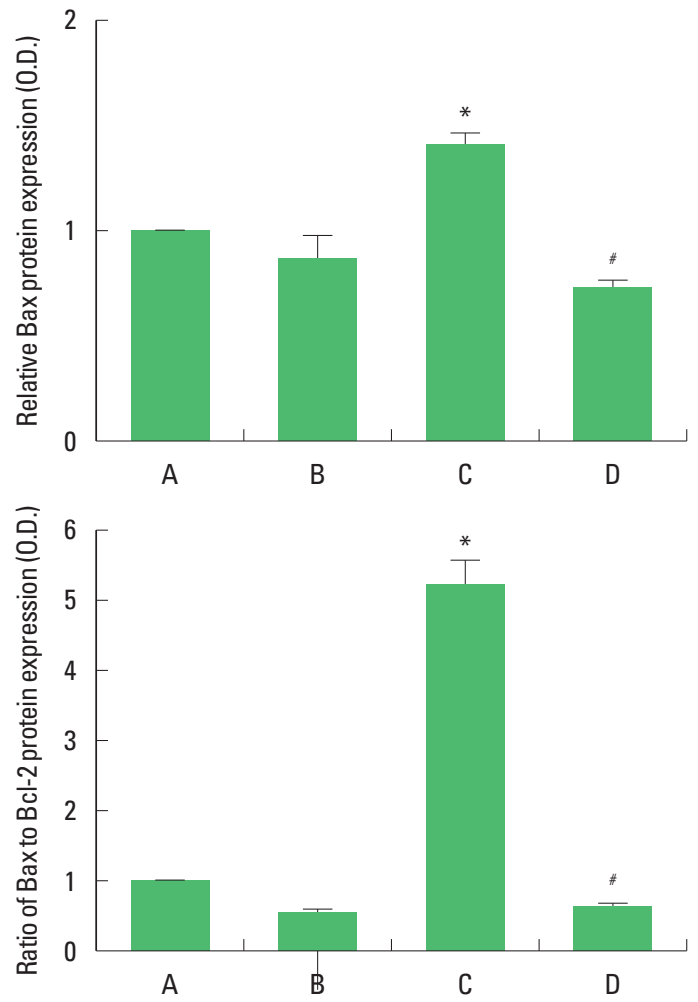

Fig. 4. Bcl-2 related X protein (Bax) and B-cell lymphoma 2 (Bcl-2) expression in the hippocampus. Left upper panel: representative expression of Bax and Bcl-2. Right upper panel: relative expression of Bax. Left lower panel: relative expression of Bcl-2. Right lower panel: ratio of Bax to Bcl-2. A, pups born to old mother rats; $B$, pups born to old mother rats with exercise; $C$, pups born to old and obese mother rats; $D$, pups born to old and obese mother rats with exercise. ${ }^{*} P<0.05$ compared with pups born to old mother rats. ${ }^{*} P<0.05$ compared with pups born to old and obese mother rats. 

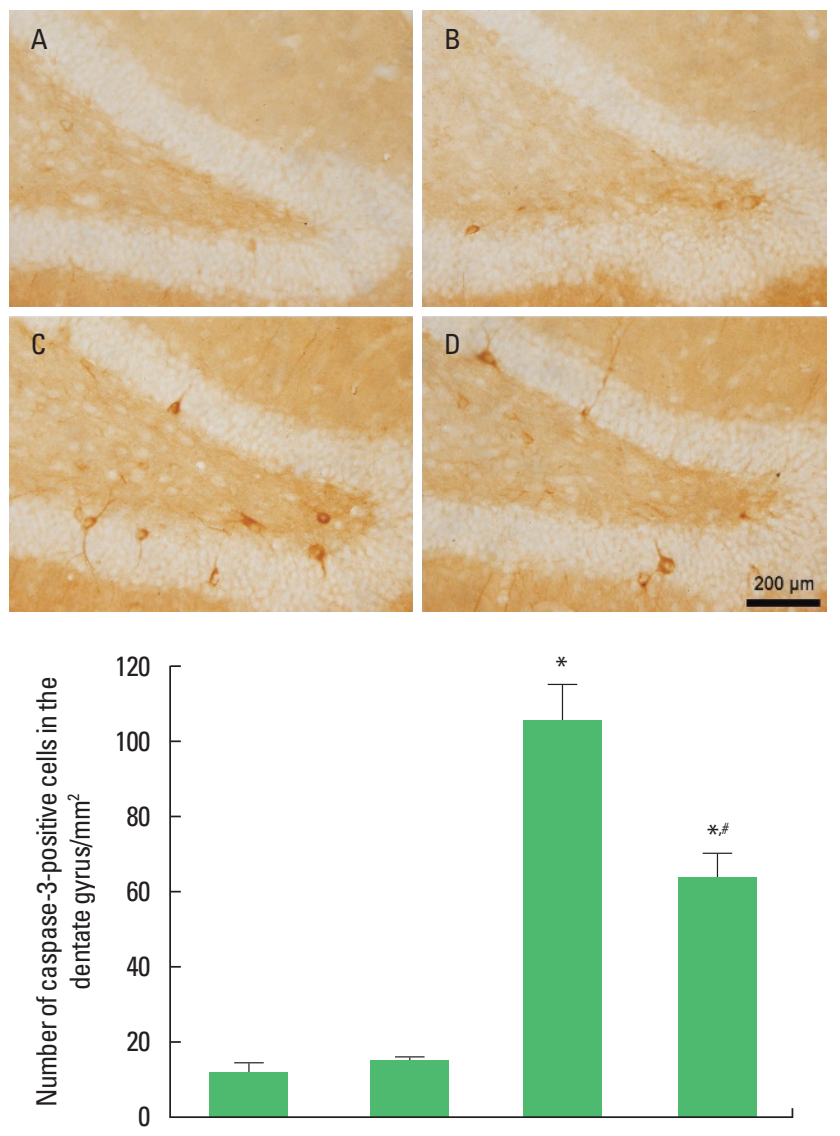

A

B

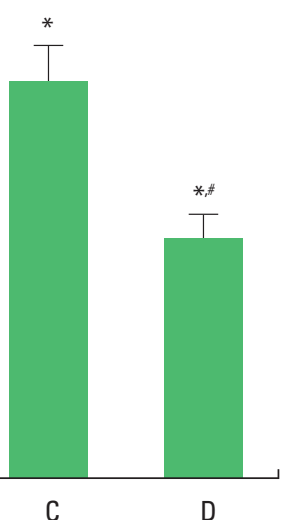

Fig. 5. Caspase-3 expression in the hippocampal dentate gyrus. Upper panel: photomicrographs of caspase-3-positive cells in immunohistochemistry. Lower panel: number of caspase-3-positive cells. A, pups born to old mother rats; $B$, pups born to old mother rats with exercise; $C$, pups born to old and obese mother rats; $\mathrm{D}$, pups born to old and obese mother rats with exercise. ${ }^{*} P<0.05$ compared with pups born to old mother rats. ${ }^{\sharp} P<0.05$ compared with pups born to old and obese mother rats.

\section{Caspase-3 immunohistochemistry}

The number of caspase-3-positive cells in the hippocampal dentate gyrus was increased in the pups born to old and obese mother rats compared to the pups born to old mother rats. In contrast, pups born to old and obese mother rats with exercise showed suppressed caspase-3-positive cell number (Fig. 5).

\section{MMP-9 expression}

Expression of MMP-9 in the hippocampus was increased in the pups born to old and obese mother rats compared to the pups born to old mother rats. In contrast, pups born to old and obese mother rats with exercise showed decreased MMP-9 expression (Fig. 6).

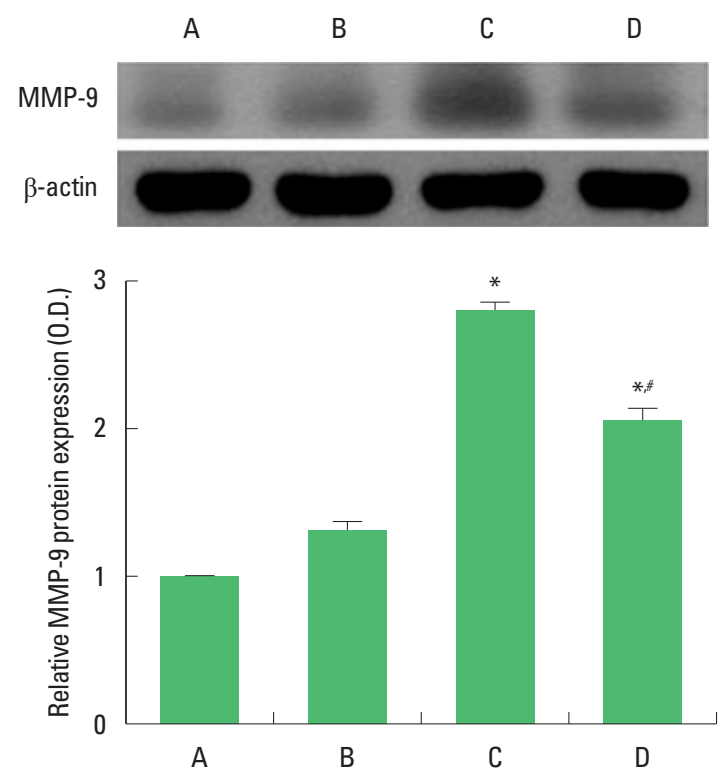

Fig. 6. Matrix metallopeptidase (MMP)-9 expression in the hippocampus. Upper panel: representative expression of MMP-9. Lower panel: relative expression of MMP-9. A, pups born to old mother rats; $B$, pups born to old mother rats with exercise; $C$, pups born to old and obese mother rats; $D$, pups born to old and obese mother rats with exercise. ${ }^{*} P<0.05$ compared with pups born to old mother rats. ${ }^{\#} P<0.05$ compared with pups born to old and obese mother rats.

\section{DISCUSSION}

Memory exercise, healthy eating, fitness and stress reduction can improve cognitive function and brain efficiency (Small et al., 2006). Spatial learning memory was aggravated by traumatic brain injury, but treadmill exercise improved memory impairment due to traumatic brain injury through upregulation of dopamine level and downregulation of dopamine receptor expression (Ko et al., 2019). In this result, spatial learning memory was decreased in the pups born to old and obese mother rats, however exercise of mother rats ameliorated spatial learning memory impairment of the pups born to old and obese mother rats. These results mean that maternal exercise improved spatial learning memory in the rat pups born to old and obese mother rats.

Enhanced secretion of proinflammatory cytokines (including TNF- $\alpha$, IL-1 $\beta$, and IL- 6 ) attributed to the damaging effect of indomethacin on gastric mucosa, and reduction of proinflammatory cytokines promoted recovery of gastric injury (Ko et al., 2020). In this result, the secretion of proinflammatory cytokines, TNF- $\alpha$, IL- 6 , IL- $1 \beta$, was increased in the pups born to old and obese mother rats, however exercise of mother rats suppressed the production of proinflammatory cytokines of the pups born to old and obese 
mother rats. These results mean that maternal exercise suppressed exacerbation of inflammation in the rat pups born to old and obese mother rats.

$\mathrm{NF}-\mathrm{KB}$ is consistently active in arthritis, asthma, atherosclerosis, gastritis, inflammatory bowel disease, sepsis, and others (Monaco et al., 2004). In obesity and aging, NF- $\kappa B$ was highly expressed and anti-inflammatory level was reduced (Kauppinen et

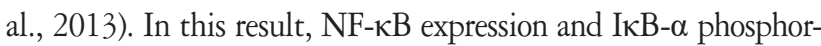
ylation were increased in the pups born to old and obese mother rats, however exercise of mother rats suppressed NF- $\mathrm{kB}$ expression and IкB- $\alpha$ phosphorylation of the pups born to old and obese mother rats. These results mean that maternal exercise suppressed inflammation inducer in the rat pups born to old and obese mother rats

Overexpressing Bax represents initiation of apoptosis (Lee et al., 2020; Song et al., 2018). Upon activation of Bax, apoptosis resumes with the consumption of Bcl-2 in complex with Bax (Dlugosz et al., 2006). Expression of Bax was enhanced, while expression of $\mathrm{Bcl}-2$ was suppressed in the old rats that underwent social isolation, which facilitated apoptosis. Meanwhile, swimming inhibited Bax expression and increased Bcl-2 expression, resulting in suppression of apoptosis (Park et al., 2020). In this result, Bax expression was enhanced and $\mathrm{Bcl}-2$ expression was suppressed, resulting in Bax to Bcl-2 ratio was increased in the pups born to old and obese mother rats. However, exercise of mother rats decreased Bax expression and increased $\mathrm{Bcl}-2$ expression, resulting in Bax to Bcl-2 ratio was suppressed in the pups born to old and obese mother rats. These results mean that maternal exercise exerted suppressive tendency on apoptosis in the rat pups born to old and obese mother rats

Caspase- 3 is one of the caspases responsible for the final trigger of apoptosis (Lee et al., 2020). Expression of caspase-3 in the hippocampus was increased in the rats of the social isolation group compared to the control rats. However, treadmill exercise decreased caspase- 3 expression in the social isolation rats (Song et al., 2018). In this result, the number of caspase- 3 was increased in the pups born to old and obese mother rats, however exercise of mother rats suppressed the number of caspase- 3 of the pups born to old and obese mother rats. These results mean that maternal exercise inhibited apoptosis in the rat pups born to old and obese mother rats.

MMP-9 is implicated in the development of aortic aneurysms and doxycycline inhibits aortic aneurysm growth through inhibition of MMP-9 (Lindeman et al., 2009). MMP-9 level was increased with the progression of idiopathic atrial fibrillation (Li et al., 2014).
MMP-9 plays a pivotal role in angiogenesis, stromal remodeling, and metastasis (Farina and Mackay, 2014). In this result, the level of MMP-9 was increased in the pups born to old and obese mother rats, however exercise of mother rats suppressed the level of MMP-9 of the pups born to old and obese mother rats. These results mean that maternal exercise inhibited inflammatory reaction in the rat pups born to old and obese mother rats

In this study, the spatial learning memory of pups born to old mother rats was not significantly affected by the maternal exercise. However, the spatial learning memory of pups born to old and obese mother rats was significantly affected. Spatial learning memory was impaired through NF- $\kappa \mathrm{B}$ activation in pups born to old and obese mother rats. However, when exercise of mother was performed, the impairment of spatial learning memory in pups was ameliorated. Therefore, it can be seen that exercise during pregnancy of older and obese mothers is an important factor in fetal health management.

\section{CONFLICT OF INTEREST}

No potential conflict of interest relevant to this article was reported.

\section{ACKNOWLEDGMENTS}

This work was supported by the Ministry of Education of the Republic of Korea and the National Research Foundation of Korea (NRF-2019S1A5A2A01040104).

\section{REFERENCES}

Albensi BC, Mattson MP. Evidence for the involvement of TNF and NF«B in hippocampal synaptic plasticity. Synapse 2000;35:151-159.

Chang GQ, Gaysinskaya V, Karatayev O, Leibowitz SF. Maternal high-fat diet and fetal programming: increased proliferation of hypothalamic peptide-producing neurons that increase risk for overeating and obesity. J Neurosci 2008;28:12107-12119.

Dlugosz PJ, Billen LP, Annis MG, Zhu W, Zhang Z, Lin J, Leber B, Andrews DW. Bcl-2 changes conformation to inhibit Bax oligomerization. EMBO J 2006;25:2287-2296.

Farina AR, Mackay AR. Gelatinase B/MMP-9 in tumour pathogenesis and progression. Cancers (Basel) 2014;6:240-296

Hwang L, Ko IG, Jin JJ, Kim SH, Kim CJ, Chang B, Rho JH, Moon EJ, Yi JW. Dexmedetomidine ameliorates memory impairment in sleep-deprived mice. Anim Cells Syst (Seoul) 2019;23:371-379. 
Jeong HY, Choi YS, Lee JK, Lee BJ, Kim WK, Kang H. Anti-inflammatory activity of citric acid-treated wheat germ extract in lipopolysaccharidestimulated macrophages. Nutrients 2017;9:730.

Kauppinen A, Suuronen T, Ojala J, Kaarniranta K, Salminen A. Antagonistic crosstalk between NF- $\mathrm{B}$ and SIRT1 in the regulation of inflammation and metabolic disorders. Cell Signal 2013;25:1939-1948.

Ko IG, Jin JJ, Hwang L, Kim SH, Kim CJ, Han JH, Kwak MS, Yoon JY, Jeon JW. Evaluating the mucoprotective effect of polydeoxyribonucleotide against indomethacin-induced gastropathy via the MAPK/NF- $\mathrm{kB}$ signaling pathway in rats. Eur J Pharmacol 2020;874:172952.

Ko IG, Kim CJ, Kim H. Treadmill exercise improves memory by up-regulating dopamine and down-regulating D2 dopamine receptor in traumatic brain injury rats. J Exerc Rehabil 2019;15:504-511.

Kuwana T, Newmeyer DD. Bcl-2-family proteins and the role of mitochondria in apoptosis. Curr Opin Cell Biol 2003;15:691-699.

Lee SS, Kim CJ, Shin MS, Lim BV. Treadmill exercise ameliorates memory impairment through ERK-Akt-CREB-BDNF signaling pathway in cerebral ischemia gerbils. J Exerc Rehabil 2020;16:49-57.

Li M, Yang G, Xie B, Babu K, Huang C. Changes in matrix metalloproteinase-9 levels during progression of atrial fibrillation. J Int Med Res 2014;42:224-230

Lindeman JH, Abdul-Hussien H, van Bockel JH, Wolterbeek R, Kleemann R. Clinical trial of doxycycline for matrix metalloproteinase-9 inhibition in patients with an abdominal aneurysm: doxycycline selectively depletes aortic wall neutrophils and cytotoxic T cells. Circulation 2009; 119:2209-2216.

Meffert MK, Chang JM, Wiltgen BJ, Fanselow MS, Baltimore D. NF-кB functions in synaptic signaling and behavior. Nat Neurosci 2003;6: 1072-1078.
Monaco C, Andreakos E, Kiriakidis S, Mauri C, Bicknell C, Foxwell B, Cheshire N, Paleolog E, Feldmann M. Canonical pathway of nuclear factor $\kappa \mathrm{B}$ activation selectively regulates proinflammatory and prothrombotic responses in human atherosclerosis. Proc Natl Acad Sci U S A 2004;101:5634-5639.

Opdenakker G, Van den Steen PE, Dubois B, Nelissen I, Van Coillie E, Masure S, Proost P, Van Damme J. Gelatinase B functions as regulator and effector in leukocyte biology. J Leukoc Biol 2001;69:851-859.

Park SS, Park HS, Kim TW, Lee SJ. Effects of swimming exercise on social isolation-induced memory impairment and apoptosis in old rats. J Exerc Rehabil 2020;16:234-241.

Small GW, Silverman DH, Siddarth P, Ercoli LM, Miller KJ, Lavretsky H, Wright BC, Bookheimer SY, Barrio JR, Phelps ME. Effects of a 14-day healthy longevity lifestyle program on cognition and brain function. Am J Geriatr Psychiatry 2006;14:538-545.

Song SH, Jee YS, Ko IG, Lee SW, Sim YJ, Kim DY, Lee SJ, Cho YS. Treadmill exercise and wheel exercise improve motor function by suppressing apoptotic neuronal cell death in brain inflammation rats. J Exerc Rehabil 2018;14:911-919.

Upadhyay D, Panduri V, Ghio A, Kamp DW. Particulate matter induces alveolar epithelial cell DNA damage and apoptosis: role of free radicals and the mitochondria. Am J Respir Cell Mol Biol 2003;29:180-187.

Vandooren J, Van den Steen PE, Opdenakker G. Biochemistry and molecular biology of gelatinase B or matrix metalloproteinase-9 (MMP-9): the next decade. Crit Rev Biochem Mol Biol 2013;48:222-272.

Wang J, Tsirka SE. Neuroprotection by inhibition of matrix metalloproteinases in a mouse model of intracerebral haemorrhage. Brain 2005; 128(Pt 7):1622-1633. 